\title{
Pier Francesco Rambaldi: Whole-body FDG PET Imaging in Oncology_Clinical Reports
}

\author{
Springer Verlag Italia, Milan 2014, ISBN: 978-88-4705294-9
}

\author{
Vania Mallardo
}

Published online: 8 August 2014

(C) Springer-Verlag Berlin Heidelberg 2014

This casebook/atlas has been written by Pier Francesco Rambaldi, Assistant Professor at the Second University of Naples, in collaboration with Giovanni Fontanella. The manual of 352 pages, enriched by 256 illustrations, almost all in colour, is structured as a collection of clinical oncology cases in which the integration of PET/CT with FDG to the classic diagnostic algorithm has been proposed, including standard radiological and laboratory texts.

The volume is divided into 12 sessions, analysing cases concerning gall bladder and bile ducts, head and neck, colon and rectum, oesophagus, gynaecology, lymphomas and thymomas, breast, melanoma, pancreas, lung, stomach and urinary tract. In each session, interesting case reports are structured as individual chapters.

The starting point of each case is represented by the detailed description of the clinical history, including previous diagnostic data and ongoing therapy. Then the role of PET/CT (in staging, restaging, prognostic evaluation, response to therapy) is considered on the basis of its capability to answer the diagnostic question made by the clinician.

The answer is widely expressed in a report in which the information obtained is described, individuating areas with
FDG uptake and their distribution, with the major goal of defining the metabolic activity of the neoplasm. The report is written as clearly and understandably as possible to serve as the communication tool for the patient and the medical prescriber. Each clinical case, supported by an extensive iconographic collection, ends with conclusions and key points. In most of the pictures, coloured arrows help to correctly indicate to the reader the anatomical area and/or relevant issues. With the same purpose, drawings better explaining some pictures, more difficult to be understood, are also occasionally presented .

In conclusion, this book provides to students and experts in nuclear medicine, radiology and oncology, but also to all other clinicians interested in better understanding the clinical role of PET/FDG in oncology, a guidance for the interpretation of the images, which correlate anatomical and functional data. In this way, a clinically valuable report correctly answering the diagnostic query is obtained. This final result may be useful for all physicians who are in charge of oncological patients to contextualize, explain and communicate the results obtained with PET/CT, which may have a dramatic impact on prognosis, choice of treatment and the quality of life not only of the patient but also of their family members.

V. Mallardo $(\bowtie)$ 